\title{
A CROSS-SECTIONAL SURVEY ON COPING BEHAVIOR OF PAKISTANI PARENTS WITH A CHILD DIAGNOSED AUTISM SPECTRUM DISORDER
}

\section{ABSTRACT}

\section{BACKGROUND AND AIMS}

Number of studies reported that most of the parents of children with developmental disabilities such as Autism spectrum disorder (ASD) are suffering from stress, anger, guilt and depression Either they not aware of the coping strategies or are in denial with self-condition, this led to adverse effects on parent's quality of life, relationship with each other and children. This study aims to discover the coping strategy that parents are following to overcome their emotional disturbance for being parent of child with ASD.

\section{METHODOLOGY}

A cross-sectional survey was responded by parents of children with ASD, enrolled via convenient sampling technique. The data was collected through a standardized questionnaire BREF-COPE, formulated in Google Docs, distributed via email or WhatsApp group.

\section{RESULTS}

A total number of 150 participants were enrolled in the study that included $74.2 \%$ of as primary care givers (mean age: $32.2 \pm 10.6$ years) and their respective autistic children (mean age: $7.1 \pm 4.4$ years). Results revealed $51.6 \%$ parents usually concentrate on their efforts with their children while $64.5 \%$ understand the reality of situation. Surprisingly, $12.9 \%$ consume alcohol or take antidepressant drugs while majority seeks emotional support from others to make them better. Overall, only $16.1 \%$ are able to implement coping strategies in their routines.

\section{CONCLUSION}

It was concluded that there is an intense need to introduce parent support group for better catharsis and inculcation of positive coping strategies among parents of children diagnosed with ASD

\section{KEYWORDS}

Autism Spectrum Disorder, developmental disability, coping behavior, emotional disturbance, Denial, catharsis
Arwa Quaid

Occupational Therapist

Thinkmatic

Arwaquaid9@gmail.com

[Quaid A. A Cross-Sectional Survey on Coping Behavior of Pakistani Parents with a Child Diagnosed Autism Spectrum Disorder Pak. j. rehabil. 2021;10(1):68-75]

DOI:10.36283/pjr.zu.10.1/0 10 


\section{INTRODUCTION}

Autism or Autism Spectrum Disorder is characterized by a neurodevelopment disorder on the basis of continuing deficits in social interaction alongside restricted and repetitive behavioral patterns or interests ${ }^{1}$. However, it can be manifested by two prototypically inflexible behaviors that can be longlasting ${ }^{2}$. Therefore, each autistic individual is different. Thus, it is described as a 'spectrum'. Nevertheless, children with autism have a number of characteristics that include attitudes, social interactions, leisure, and play so these characteristics may seem to be more of a trend ${ }^{3}$.

Autism is usually associated with physical development and psychological state of an individual. ${ }^{4}$. These individuals are frequently exposed to social challenges that include lack of respect, lack of understanding and lack of support from others. These challenges hinder them to be an integral part in a non-autistic world due to which parents or guardians are facing several struggles with their children $^{5}$. Of struggling challenges that parents usually face, they include children's challenges in educational institutes, social stigma and suppression, stereotypes that are subsequent in children with Autism Spectrum Disorder ${ }^{6}$. Since stigma and stereotyping are very common issues with these children, it is very difficult for parents to adore it without failing to collapse and face society but rather consider easier to stigmatize. Furthermore, society intensifies and facilitates the stigma due to which it is very difficult for parents to face the situation ${ }^{7}$. Moreover, autistic children have behavioral tendencies which are quite hard for the parents to manage because of their outburst actions, self-stimulatory behaviors, lack of sleeping etc. through which parents suffer and make their self-esteem questionable while resulting isolation from the society ${ }^{8}$.
Nevertheless, a number of parents showed significant success in nowemerging coping strategies that had led them to acquire positivity in their lives ${ }^{9}$.

The signs and symptoms that are linked with autism spectrum disorder are variable and unpredictable. Moreover, these symptoms have a long-lasting nature as well as being difficult for parents to care of these symptoms in their children especially during early ages of development ${ }^{10}$. Moreover, caregiver's limitations towards managing autistic children may result from difficulty in gathering information and attaining early intervention services ${ }^{11}$. Later on, significant stressors can arise with the child's maturity related to the planning of the estate, finances, and care. In particular, lower family well-being may cause a bidirectional, negative cycle that will exacerbate the behavioral problem of the child as well as reduce the positive effects of therapeutic interventions ${ }^{12}$. A recent report investigated the impact of coping mechanisms and social support upon behavioral symptoms of autistic children. This report included sixty-five mothers of children with autism spectrum disorder that revealed certain disengagement and distractions in implementing coping strategies that may lead to poor well-being. Furthermore, the study showed that cognitive reframing that may lead to better levels of wellbeing. On the other hand, a substantial correlation between behavioral problems with children and maternal well-being was found out ${ }^{13}$. Another study implemented the actor-partner interdependence model to examine the predictors of relationship satisfaction between parents and autistic children. The study revealed positive attributes of the parent resulting in a higher consummation of the parental-child relationship. Moreover, recognizing the parent's advantages using social and emotional support, and perceiving social support from their spouse may also result 
in satisfaction with the relationship. In addition, concentrating on positive factors may enhance the consistency of the relationships ${ }^{14}$.

Another study explained the role of coping mechanisms and perceptions of self-efficacy as predictors of life satisfaction in parents of autistic children showed higher expressing emotions and strategies of social support in mothers particularly. These effective coping mechanisms and a degree of self-efficacy play a significant role in developing effective management for ASD children. It was also showed that the need for early intervention with parents to promote coping strategies plays a vital role in curing autism ${ }^{15}$. A study was conducted to compare parental stress with social support and coping strategies in families of children with low and high functioning autism suggested the desirability of fostering functional coping strategies particularly in those with children with low functioning autism ${ }^{16}$.

Similarly, two researchers sponsored mindfulness-based interventions to enhance the quality of life in different communities among parents of children with autism. They demonstrated significant improvements in psychological health domain measures. The MBI tool used in the study was found to be a culturally adaptable and successful tool for improving the quality of life in autistic child parents ${ }^{17}$. As parents of autistic children face challenges due to day-to-day stressors associated with their child's disorder due to certain characteristics may influence how they approach stressful events in life and potentially help them cope with some of the deleterious effects of extreme stress ${ }^{18}$.Therefore, it is substantial for the caregivers to identify the challenges and approaches that autistic children have embraced and implement certain coping strategies for the effective management. Thus, this study is aimed to assess the coping behavior of parents with Autism Spectrum Disorder children to rule out the ongoing challenges between parent-child relationships. Another study attempted to assess the comparison between parents who have children suffering from autisms spectrum disorder that presumably have increased stress level with decreased well-being than those parents whose children are normally developing ones. Therefore, this study assessed stress levels of parents who either had their child diagnosed with autism spectrum disorder or not. Hence, thirty-seven adult parents were taken whose children had a current history of ASD while forty-one parents were taken whose children had no current or past history of ASD. Eventually, the results concluded with increased stress levels of parents whose children were diagnosed with current $\mathrm{ASD}^{19}$. There is a different study that compared different physiological measures of stress that included ambulatory blood pressure, heart rate, alpha-amylase and cortisol in blood between equal number of parents having either children with ASD or not being ASD. Of these parents, saliva samples were collected to measure different previously mentioned blood components that determined stress levels and held blood pressure monitor for a day. The results revealed a surprising outcome. Both groups of parents whose children had either ASD or not had no significant differences in the levels of stress determiners in their saliva. However, only significant difference was found in cortisol levels after 30 minutes of waking up. Interestingly, parents with ASD children who were assumed to have increased levels of stress determiners had reported to follow coping strategies that included emotional support ${ }^{20}$. There is a supportive study that further proves coping strategies to be effective upon reducing stress levels of parents whose children are suffering from ASD. Hence, a study assessed relationship between coping 
strategies and stress levels among parents whose children were suffering from ASD. Level of coping strategies implementation was assessed with the help of Coping Health Inventory for Parents Questionnaire while stress levels were measure over Parental Stress Scale. A prevalence of $57.7 \%$ of coping strategies among these parents were found while $76 \%$ of them had moderate stress levels. Spearman's Rank correlation test revealed a negative association of coping strategies with stress levels of these parents $(r=$ $0.699)^{21}$.

The study aims to reveal the coping behavior and the need of it among parents that enables to encounter the stress induced due to the diagnosis of their children with ASD

\section{METHODOLOGY}

Study Setting: Data was collected from Parents whose children were enrolled in different rehabilitation institutes in Pakistan

Target Population: Parents of children with Autism Spectrum Disorder.

Study Design: Cross-sectional survey.

Duration of Study: 6-8 months.

Sampling Technique: Non-Probability Convenience Sampling Technique. Sample size was calculated by using Open EPI software. Therefore, at anticipated frequency of $50 \%$, with Confidence Interval of $95 \%$ with margin of error at $5 \%$, the sample size was $n=150$

\section{Sample Selection} Inclusion criteria

- $\quad$ Parents with ASD children of any age who have enrolled for $>6$ months in rehabilitation.

\section{Exclusion criteria}

- $\quad$ Parents of Children with ASD along with severe physical or intellectual complications.

- $\quad$ Parents of children with ASD enrolled in rehabilitation program but with history of current smoking.
- $\quad$ Parents of children with ASD enrolled in rehabilitation programs with neurodegenerative diseases.

Data Collection Tool and Procedure: A standardized questionnaire BRIEF-COPE was used for the assessment of the coping skills of parents. A number of 60 questions on a scale of 4 i.e. (1) I haven't been doing this at all, (2) I've been doing this a little bit, (3) I've been doing this a medium amount (4) I've been doing this a lot were used to record the responses that determine the coping ability of each parent in different situations.

Participants were enrolled in study through convenience sampling technique from different rehabilitation institutes. The questionnaire was formulated in Google Docs and sent to participants via email or WhatsApp group. Prior to collection, all participants were provided an understanding about the questionnaire in order to record the responses accurately.

Data was analyzed on Google Docs Editor. The demographic characteristics of the participants were represented through frequency, mean and standard deviations whereas the participant responses were evaluated through frequency and percentage.

Ethical consideration was taken via verbal and written consent by the participant before starting the data collection. All information of the participants will be kept anonymous under investigator's supervision. 


\section{RESULTS}

A total number of 150 participants enrolled in the study representing majority of mothers (74.2\%) as primary care givers with mean age of $32.2 \pm 10.6$ while all parents reported to have autistic children with mean age of 7.1 \pm 4.4 . However, only one parents reported to have two children with autism. Number of parents (51.6\%) answered that they usually concentrate on their efforts with children, (48.4\%) substitute activities to take their mind off thing while (64.5\%) understand the reality of situation. Surprisingly, (12.9\%) using little bit alcohol or drugs to make them better and majorly seek emotional support from others as shown in Figure-1

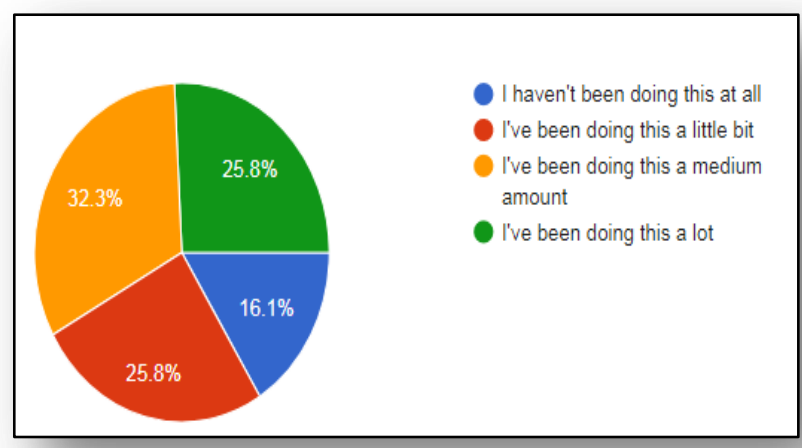

Figure-1 shows percentage of parents getting emotional support from others

Besides, (38.7\%) trying to deal with the situation and (64.5\%) trying to make the situation better and face the real situation (58.1\%). However, (50\%) answered that they have been saying things to let their unpleasant feelings escape and (46.7\%) reported to get help and advice from others as shown in Figure-2.

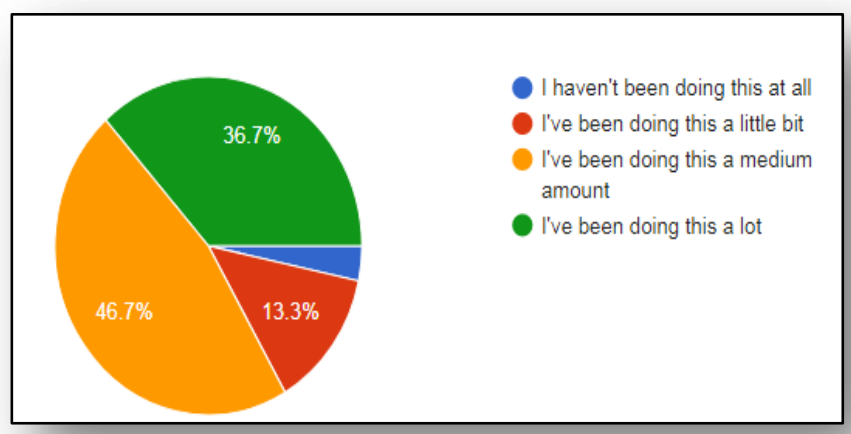

Figure-2 shows percentage of parents getting help and advice from other people

It has been revealed that (41.9\%) parents are trying to be more positive while (29\%) seem to do little criticism to them. However, (41.9\%) get comfort and understanding from someone to cope up although, only (16.1\%) are found to attempt coping a lot, (32.3\%) little bit and (29\%) haven't been doing this at all as shown in Figure-3.

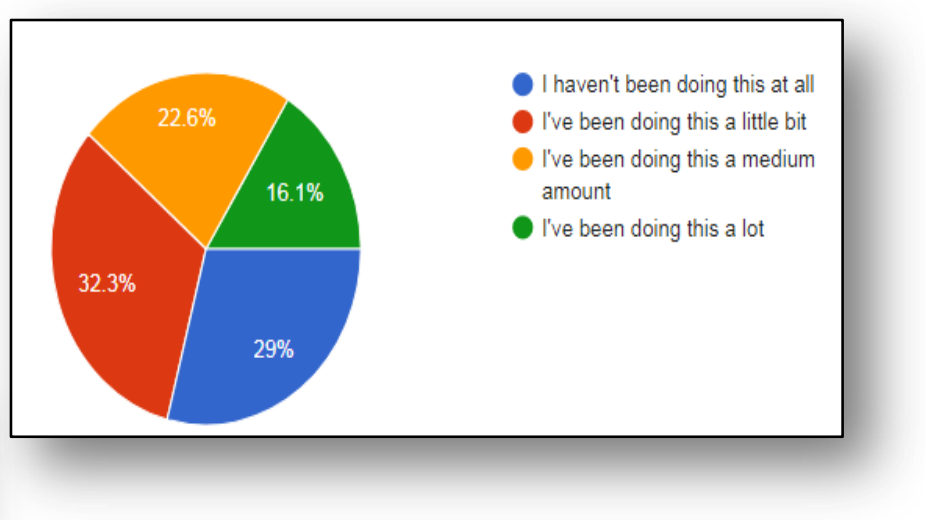

Figure-3 shows percentage of parents giving up the attempt to cope

A number of (51.7\%) participant reported to look for something good in what is happening, (16.7\%) have been doing something to think about the situation less as shown in Figure-4 and (56.7\%) has accepted the reality of fact about the situation.

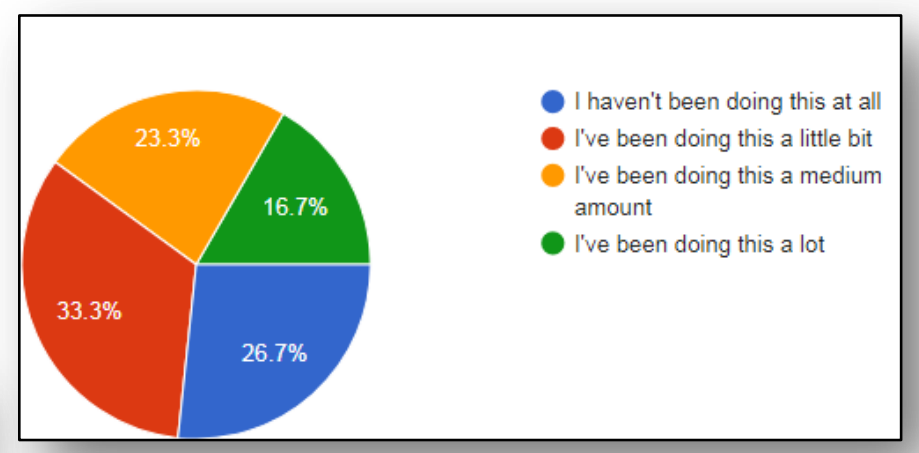

Figure- 4 shows percentage of parents doing something to think about it less such as going to movies, watching TV, reading or shopping 


\section{DISCUSSION}

The findings of this study showed that number of parents (51.6\%) usually concentrate on their efforts with children, (48.4\%) substitute activities to take their mind off thing while (64.5\%) understand the reality of situation. Surprisingly, (12.9\%) are using little bit of alcohol or drugs to make them feel better while majority seeks emotional support from others. On the other hand, $(38.7 \%)$ try to deal with the situation and (64.5\%) try to make the situation better and face the real situation (58.1\%). However, (50\%) answered that they had been out bursting while saying things to let their unpleasant feelings escape and (46.7\%) reported to get help and advice from others. However, (41.9\%) get comfortable with counseling and understanding from someone to cope up though only (16.1\%) are found to attempt coping a lot. The findings of the study showed that primary care givers face several barriers with their coping skills because of emotional support from others that may lead to criticism. Moreover, prayers and meditation play a significant role in a parent's life, especially in mothers. The stigma of having a special child intimates some parents to take drugs and blame themselves that further affects their quality of life resulting child negligence from their end. Some studies demonstrated that men have higher coping skills than women. Furthermore, the caregivers observed for consolation and support from companions, in the form of casual or formal help from different programs.

Autism is a complex developmental condition that leads to impairments in social, emotional, communication, and repetitive behavior impairments ${ }^{22}$. The characteristics lead to cause a difference in every ASD child. A study conducted by Prata et al $^{23}$ reported high-stress levels in ASD children related to factors influencing including family, professional, economic, and social support. Similarly, in a local study, a moderate level of stress was reported in parents of autistic children ${ }^{24}$. Moreover, studies have reported that a number of special children seem to be related to increased parental stress. Moreover, a study conducted by Gupta et $\mathrm{al}^{25}$ reported that female autistic child causes more parental-child dysfunction, also the parents that have prestigious and lucrative jobs had more stress. Besides, a number of parents turn towards God as a coping strategy when they see disability as the will of God.

A number of upfront estimations were noted from the caretakers of children with ASD taming caregivers towards sound therapy approaches to deliver through the skills required to address events in direction to raise the parent's self-confidence as a critical part of the health practice in order to promote better health outcomes ${ }^{26}$. Consecutively, this study exposes the resiliency and extremely adaptive nature of caregivers who stand under severe strain and stress of caring for a child with autism. Moreover, the active ways managed as a family were in the areas of informal and formal social support systems where the applicants' used inactive judgment to cope up.

This study highlights significant findings regarding coping strategies attempted by parents with autistic children. Moreover, a standardized questionnaire was used to evaluate the coping outcomes.

Several limitations occurred in this study such that a small sample size was not sufficient to generalize the results. In addition, the author found difficulty in participants' recruitment amid COVID-19 pandemic. Furthermore, the factors associated to coping in parent-child relationship were not evaluated. 
Besides survey-based studies, number of interventions must be applied to examine the behavioral patterns in parent-child relationship in order to determine the coping outcome, comparatively on a large sample size to generalize the results.

\section{CONCLUSION}

It was concluded that majority of participants has been trying to cope up by accepting the reality, soundness of situation or seek emotional support from others related to their children. However, few were using little bit alcohol or drugs to make them better. Therefore, multicenter trials should be conducted to evaluate the factors associated with coping skills to effectively implement the strategy to promote healthy relationships. Moreover, a parent support group is a desperate need for parent's mental health and their emotional support in order to have healthy relationship with their children.

\section{REFERENCE}

[1] Samson AC, Phillips JM, Parker KJ, Shah S, Gross JJ, Hardan AY. Emotion dysregulation and the core features of autism spectrum disorder. Journal of Autism and developmental Disorders. 2014 Jul 1;44(7):1766-72.

[2] Ha S, Sohn IJ, Kim N, Sim HJ, Cheon KA. Characteristics of brains in autism spectrum disorder: structure, function and connectivity across the lifespan. Experimental neurobiology. $2015 \mathrm{Dec}$ 1;24(4):273-84.

[3] Anaby D, Hand C, Bradley L, DiRezze B, Forhan M, DiGiacomo A, Law M. The effect of the environment on participation of children and youth with disabilities: a scoping review. Disability and rehabilitation. 2013 Sep 1;35(19):1589-98.

[4] Lord C, Brugha TS, Charman T, Cusack J, Dumas G, Frazier T, Jones EJ, Jones RM, Pickles A, State MW, Taylor JL. Autism spectrum disorder. Nature reviews Disease primers. 2020 Jan 16;6(1):1-23.

[5] Russell S, McCloskey CR. Parent perceptions of care received by children with an autism spectrum disorder. Journal of Pediatric Nursing. 2016 Jan 1;31(1):21-31.

[6] Makin C, Hill V, Pellicano E. The primary-to-secondary school transition for children on the autism spectrum: A multi-informant mixedmethods study. Autism \& Developmental Language Impairments. 2017 Jan;2:2396941516684834.

[7] Gorlin JB. Autism and Society: How ASD Influences the Extended Family and Society. Systemically Treating Autism. 2019 Feb 18:68-76.

[8] Durrani H. Sensory-Based Relational Art Therapy Approach (S-BRATA): A Framework for Art Therapy With Children With ASD. Art Therapy. 2020 Feb 6:1-9.

[9] Vernhet C, Dellapiazza F, Blanc N, Cousson-Gélie $F$, Miot $S$, Roeyers $H$, Baghdadli A. Coping strategies of parents of children with autism spectrum disorder: A systematic review. European child \& adolescent psychiatry. 2019 Jun;28(6):747-58.

[10] Jones CR, Simonoff E, Baird G, Pickles A, Marsden AJ, Tregay J, Happé F, Charman T. The association between theory of mind, executive function, and the symptoms of autism spectrum disorder. Autism Research. 2018 Jan;11(1):95-109.

[11] Papadopoulos C, Lodder A, Constantinou G, Randhawa G. Systematic review of the relationship between autism stigma and informal caregiver mental health. Journal of autism and developmental disorders. 2019 Apr 1;49(4):1665-85.

[12] Piven J, Elison JT, Zylka MJ. Toward a conceptual framework for early brain and behavior development in autism. Molecular psychiatry. 2017 Oct;22(10):1385-94. 
[13] Daou N, Hady RT, Poulson CL. Teaching children with autism spectrum disorder to recognize and express emotion: A review of the literature. International Electronic Journal of Elementary Education. 2016;9(2):419-32.

[14] Ekas NV, Tidman L, Timmons L. Religiosity/Spirituality and Mental Health Outcomes in Mothers of Children with Autism Spectrum Disorder: The Mediating Role of Positive Thinking. Journal of autism and developmental disorders. 2019 Nov 1;49(11):4547-58.

[15] Salas BL, Rodríguez VY, Urbieta CT, Cuadrado $E$. The role of coping strategies and self-efficacy as predictors of life satisfaction in a sample of parents of children with autism spectrum disorder. Psicothema. 2017;29(1):55-60.

[16] Costa AP, Steffgen G, Vögele C. The role of alexithymia in parent-child interaction and in the emotional ability of children with autism spectrum disorder. Autism Research. 2019 Mar;12(3):458-68.

[17] Rayan A, Ahmad M. Psychological distress in Jordanian parents of children with autism spectrum disorder: The role of positive reappraisal coping. Archives of Psychiatric Nursing. 2017 Feb 1;31(1):38-42.

[18] Neff K, Germer C. Self-Compassion and Psychological. The Oxford handbook of compassion science. 2017 Sep 26:371.

[19] Costa AP, Steffgen G, Ferring D. Contributors to well-being and stress in parents of children with autism spectrum disorder. Research in Autism Spectrum Disorders. 2017 May 1;37:61-72.

[20] Padden C, James JE. Stress among parents of children with and without autism spectrum disorder: a comparison involving physiological indicators and parent self-reports.
Journal of developmental and Physical disabilities. 2017 Aug;29(4):567-86.

[21] Dwirexsi W, Lukman M, Rafiyah I. The correlation between coping strategy and stress of parents who have children with autism. Journal of Nursing Care. 2018 Dec 28;1(3).

[22] Sharma SR, Gonda X, Tarazi Fl. Autism spectrum disorder: classification, diagnosis and therapy. Pharmacology \& therapeutics. 2018 Oct 1;190:91104.

[23] Prata J, Lawson W, Coelho R. Parent training for parents of children on the autism spectrum: a review. Health. 2018;5:3.

[24] Reed P. Child behaviour problems moderate effectiveness of coping strategies except for reframing for mothers of children with ASD. Research in Autism Spectrum Disorders. 2020 Aug 1;76:101589. [25] Gupta N, Sachdev A, Gupta D, Gupta S, Gupta D, Nanavati J. Autism and Allergy-Are They Linked?. The Indian Journal of Pediatrics. 2018 Dec 1;85(12):1132-3. [26] Feroz R. Perspectives of Facilitators and Parents about the Development of ASD Children. 